\title{
Influence of echocardiographic and radiographic characteristics on atrial sensing amplitude in patients with Linox Smart S DX defibrillation leads
}

\author{
Marcin Michalak ${ }^{1}$, Andrzej Cacko ${ }^{1,2}$, Agnieszka Kapłon-Cieślicka ${ }^{1}$, Monika Budnik², \\ Przemysław Stolarz ${ }^{1}$, Grzegorz Opolski ${ }^{1}$, Marcin Grabowski ${ }^{1}$ \\ ${ }^{1} 11^{\text {st }}$ Chair and Department of Cardiology, Medical University of Warsaw, Warsaw, Poland \\ ${ }^{2}$ Department of Medical Informatics and Telemedicine, Medical University of Warsaw, Warsaw, Poland
}

\begin{abstract}
Background: Single-lead for implantable cardioverter-defibrillator (ICD) with floating atrial sensing dipole is a new diagnostic tool with the potential advantage in terms of arrhythmia discrimination. We sought to determine whether right heart size and dipole position influence atrial sensing.

Methods: Atrial sensing (AS) amplitude was measured during implantation (PP, periprocedural), predischarge (IHFU, in-hospital follow-up) and 3-6 months after the procedure (AFU, ambulatory follow-up). Results were related to atrial dipole position in the right atrium (RA) on the basis of chest $X$-ray examination as well as right heart dimensions at echocardiography.

Results: Twenty-four patients were included into final analysis. In 14 (58.3\%) patients, sensing dipole was located in regions 1 and 2 of the $R A$. AS amplitude was greater in regions 1 and 2 when compared to other locations (3.15 vs. $1.2 \mathrm{mV}, p=0.045,7.53 \mathrm{vs.} 3.8 \mathrm{mV}, p<0.001$ and $5.63 \mathrm{vs} .2 .44 \mathrm{mV}$, $p=0.017$ for PP measurements, IHFU and AFU, respectively). There was a significant negative correlation between AS-PP and short RA dimension (RADs) ( $r=-0.56, p=0.02), A S-I H F U$ and RA area $(R A A)(r=-0.45, p<0.05), A S-A F U$ and long RA dimension (RADl) $(r=-0.46 ; p=0.02), A S-A F U$ and RADs $(r=-0,48, p=0.02$ ), and AS-AFU and RAA (and $r=-0.52, p<0.01$ ). There was no relationship between $A S$ and other right heart dimensions.

Conclusions: Larger RA size and low sensing dipole location were associated with lower AS amplitude in single-lead dual chamber ICD. (Cardiol J 2017; 24, 6: 671-676)
\end{abstract}

Key words: implantable cardioverter-defibrillator, DX, defibrillation leads, atrial sensing

\section{Introduction}

Sudden cardiac death is one of the most common causes of death in subjects with heart failure $(\mathrm{HF})$, irrespective of its etiology [1, 2]. Introduction of implantable cardioverter-defibrillators (ICD) appeared to significantly cut mortality in this group of patients, thus implantation of ICDs became standard care in secondary and primary prevention [3, 4]. Prevalence of supraventricular tachyarrhythmias, mainly atrial fibrillation (AF), is higher among patients with $\mathrm{HF}$ and is associated with greater morbidity and mortality $[5,6] . \mathrm{AF}$ is also one of the most frequent causes of inappropriate therapy in patients with ICDs [7, 8]. Most patients enrolled into ICD studies were implanted with single-lead devices [9, 10]. However, real life data showed that many patients, although they do not need atrial pacing, are implanted with additional atrial lead which increases specificity in arrhythmia diagnosis, but on the other hand, may be associated with significant increase in the rate of periprocedural complications [11, 12] and mortality [12]. There are some studies showing that

Address for correspondence: Marcin Grabowski, MD, PhD, $1^{\text {st }}$ Chair and Department of Cardiology, Medical University of Warsaw, ul. Banacha 1a, 02-950 Warszawa, Poland, e-mail: grabm@tlen.pl

Received: 08.05.2016 Accepted: 19.07.2016 
data obtained from atrial lead may reduce rates of inappropriate therapy although most clinical trials provided mixed results in this area [13-15].

Several automatic algorithms using atrial sensing were developed to minimize the risk of inappropriate therapy [16-18]. Employment of Biotronik's DX ICD lead systems (defibrillator leads with floating atrial sensing dipole) may reduce the rate of inappropriate therapies owing to SMART ${ }^{\circledR}$ algorithm, so far available only in dual-lead ICDs.

We sought to determine whether right atrium (RA) size and/or atrial sensing (AS) rings position have an influence on atrial signal amplitude in patients with Biotronik's DX ICDs.

\section{Methods}

\section{ICD implantation}

Patients with sinus rhythm who met criteria for implantation of single-chamber ICD according to European Society of Cardiology/European Heart Rhythm Association guidelines were considered eligible for inclusion into the study. Procedures were performed by two experienced operators. The technique of ICD implantation with destination lead tip location (apex, septum or right ventricle outflow tract [RVOT]) was left for discretion of the operator. Periprocedural electrical parameters, i.a. preamplified atrial sensing amplitude (AS-PP, periprocedural atrial sensing), were measured with Medtronic Analyzer but achieving acceptable atrial signal was not mandatory if ventricular sensing and threshold was optimal. One type of defibrillation lead was used, Linox ProMRI S DX 65/15, i.e. single-coil active fixation lead with AS dipole located $15 \mathrm{~cm}$ from the lead tip. Two models of ICDs were implanted, i.e. Lumax 540 VR-T DX (patients 1-14) and Iforia 5 VR-T DX (patients 15-24), due to changes with time in models availability in our center.

\section{Radiographic assessment}

To assess AS dipole location standard chest $\mathrm{X}$-ray examination in standing position and during maximal inspiration was performed. The area of interest, i.e. RA and vena cava superior, was divided into 6 regions as showed in Figure 1. Each patient was assigned to corresponding region according to localization of point lying in the middle of distance between AS rings.

\section{Echocardiographic assessment}

Echocardiographic examination was performed using Philips iE33 system (Philips Healthcare,
Amsterdam, the Netherlands) during hospital stay associated with ICD implantation.

Assessment of the right ventricle. To assess right ventricular size we took the following steps:

- measurement of RVOT proximal and distal dimensions (in the parasternal long-axis and short-axis views);

- measurement of right ventricular basal, midcavity and longitudinal dimensions (in the apical 4-chamber view, adjusted to obtain the recommended "right ventricle-focused view"). All measurements were performed in accordance with 2010 guidelines of the American Society of Echocardiography (ASE), endorsed by the European Association of Cardiovascular Imaging (formerly European Association of Echocardiography [EAE]) and the Canadian Society of Echocardiography (CSE) [19].

Assessment of the right atrium. To assess RA size we took the following steps:

- measurement of RA major/long (RADl) and minor/short dimensions (RADs);

- measurement of RA area (RAA).

Measurements were performed in the standard apical 4-chamber view (as shown in Fig. 2), in accordance with 2010 ASE/EAE/CSE guidelines [19].

Assessment of the left ventricle. For further assessment of longitudinal heart size, we additionally measured left ventricular longitudinal dimension in the standard apical 4-chamber view [20].

\section{ICD follow-up}

Complete follow-up of ICD device with assessment of AS was conducted 2-3 days after the procedure (IHFU, in-hospital follow-up) and 3-6 months later (AFU, ambulatory follow-up) as a part of routine practice at our institution. Newer generation of ICDs used, i.e. Iforia 5 VR-T DX, has a possibility to measure the whole range of AS amplitude, while Lumax 540 VR-T DX shows maximal value of AS at $8.0 \mathrm{mV}$. To unify results AS exceeding $8.0 \mathrm{mV}$ in patients with Iforia $5 \mathrm{VR}-\mathrm{T}$ DX were reported as $8.0 \mathrm{mV}$.

All patients included into the study agreed for chest X-ray examination, echocardiographic study, and periprocedural and during follow-up ICD parameters measurements, which were part of routine practice in our center.

\section{Results}

Twenty-five consecutive patients implanted with Biotronik ICD DX were included into the study. One patient died before outpatient 


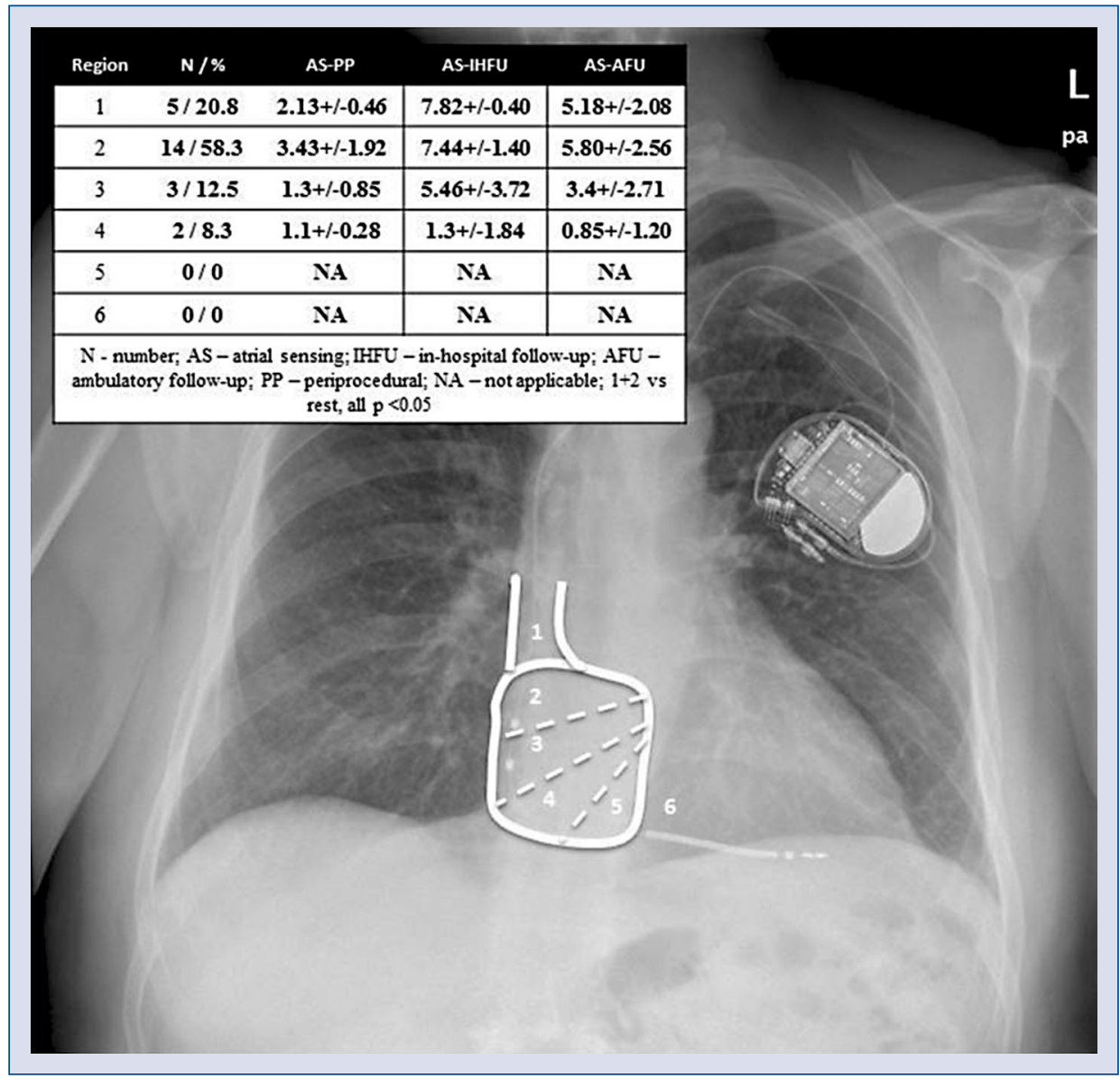

Figure 1. Assessment of the sensing dipole position in the right atrium and its influence on atrial sensing amplitude.

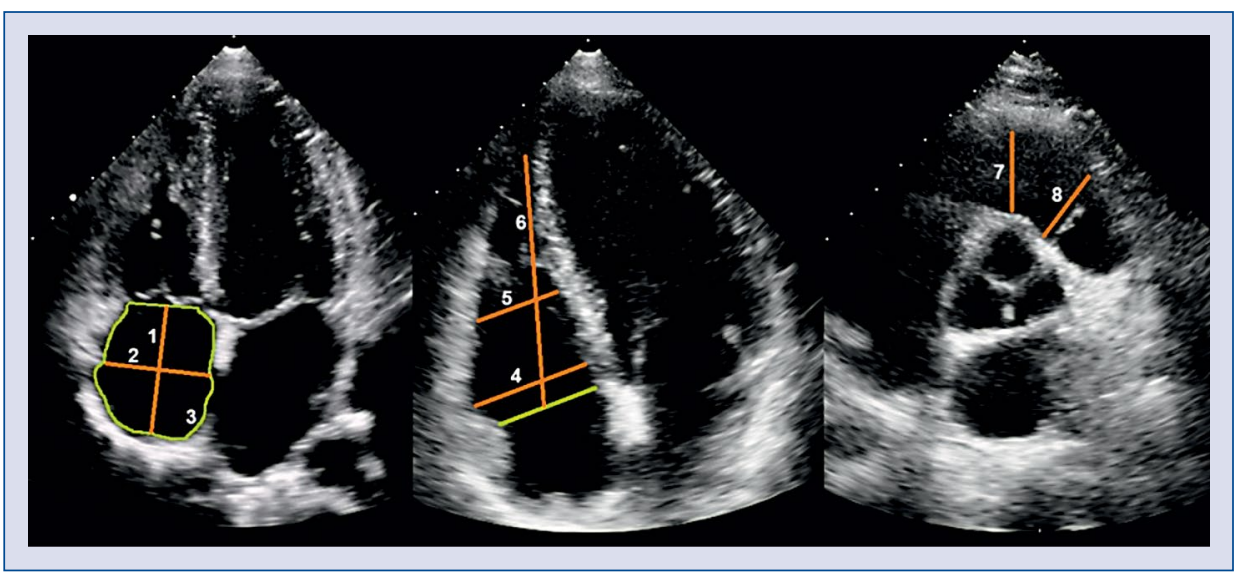

Figure 2. Echocardiographic assessment of right heart dimensions; 1 - right atrium dimension long; 2 - right atrium dimension short; 3 - right atrium area; 4 - right ventricle basal dimension; 5 - right ventricle mid-cavity dimension; 6 - right ventricle longitudinal dimension; 7 - right ventricular outflow tract proximal dimension; 8 - right ventricular outflow tract distal dimension.

follow-up and was not included into the final analysis. Baseline characteristic of study population (24 males, mean age $62 \pm 13$ years) is presented in Table 1. In 1 patient, atrial signal amplitude was below the minimal measurable value of $0.4 \mathrm{mV}$ during AFU. 
Table 1. Baseline characteristics of the study population.

\begin{tabular}{|c|c|}
\hline Age [years] & $62 \pm 13$ \\
\hline Male & $24 / 100 \%$ \\
\hline Height $[\mathrm{cm}]$ & $173 \pm 6.5$ \\
\hline Weight [kg] & $76.5 \pm 17.0$ \\
\hline \multicolumn{2}{|l|}{ Indication } \\
\hline Dilated cardiomyopathy & $8.3 \%$ \\
\hline ischemic cardiomyopathy & $91.7 \%$ \\
\hline NYHA I & $4.2 \%$ \\
\hline NYHA II & $66.6 \%$ \\
\hline NYHA III & $29.2 \%$ \\
\hline NYHA IV & $0.0 \%$ \\
\hline Primary prevention & $79.2 \%$ \\
\hline Secondary prevention & $20.8 \%$ \\
\hline \multicolumn{2}{|l|}{ Medical history } \\
\hline Hypertension & $58.3 \%$ \\
\hline Dyslipidemia & $58.3 \%$ \\
\hline CKD (eGFR < 60 mL/min) & $33.3 \%$ \\
\hline Type 2 diabetes mellitus & $33.3 \%$ \\
\hline Atrial fibrillation & $8.3 \%$ \\
\hline \multicolumn{2}{|l|}{ Atrial sensing [mV] } \\
\hline Periprocedural & $2.72 \pm 1.76$ \\
\hline In-hospital follow-up & $6.72 \pm 2.45$ \\
\hline Ambulatory follow-up & $4.97 \pm 2.75$ \\
\hline \multicolumn{2}{|l|}{ Chest X-ray } \\
\hline \multicolumn{2}{|l|}{ Dipole position: } \\
\hline 1 & $20.8 \%$ \\
\hline 2 & $58.3 \%$ \\
\hline 3 & $12.5 \%$ \\
\hline 4 & $8.3 \%$ \\
\hline 5 & $0.0 \%$ \\
\hline 6 & $0.0 \%$ \\
\hline \multicolumn{2}{|l|}{ Echocardiography } \\
\hline RADI [cm] & $4.94 \pm 0.76$ \\
\hline RADs [cm] & $4.13 \pm 0.71$ \\
\hline $\mathrm{RAA}\left[\mathrm{cm}^{2}\right]$ & $17.99 \pm 5.48$ \\
\hline RVD RVOT 1 [cm] & $3.08 \pm 0.42$ \\
\hline RVD RVOT 2 [cm] & $2.77 \pm 0.41$ \\
\hline RVD AP4C 1 [cm] & $4.04 \pm 0.69$ \\
\hline RVD AP4C 2 [cm] & $3.04 \pm 0.42$ \\
\hline RVD AP4C $3[\mathrm{~cm}]$ & $7.56 \pm 1.17$ \\
\hline LVEF [\%] & $28.00 \pm 9.39$ \\
\hline
\end{tabular}

CKD — chronic kidney disease; eGFR — estimated glomerular filtration rate; NYHA - New York Heart Association; RADL — right atrium diameter long; RADs - right atrium diameter short; RAA right atrium area; RVD — right ventricle diameter; RVOT — right ventricular outflow tract; AP4C - apical 4-chamber; LVEF — left ventricular ejection fraction
Periprocedural assessment of AS rings position was consistent with the results of assessment of follow-up chest X-ray and did not change significantly comparing two different body positions (horizontal during implantation vs vertical during X-ray examination). In most patients $(\mathrm{n}=14$; $58.3 \%$ ), AS rings were localized in region 2 and 1 $(\mathrm{n}=5 ; 28 \%)$. Details on AS rings localizations and mean atrial signal amplitude are presented in Figure 1 . We observed significantly greater AS amplitude for regions 1 and 2 when compared with other regions (in fact 3 and 4$)(3.15$ vs. $1.2, p=0.045$, 7.53 vs. $3.8, p<0.001$ and 5.63 vs. $2.44 ; \mathrm{p}=0.017$ for periprocedural measurements, IHFU and AFU, respectively).

We found significant, negative correlation of: AS-AFU and RADl ( $r=-0.46 ; p=0.02)$, AS-PP and RADs $(r=-0.56, p=0.02)$, AS-AFU and RADs $(r=-0.48, p=0.02)$, AS-IHFU and RAA $(r=-0.45$, $\mathrm{p}<0.05$ ), and AS-AFU and RAA (and $\mathrm{r}=-0.52$, $\mathrm{p}<0.01$ ). There were no other significant correlations with other echocardiographic parameters listed in Table 1.

When echocardiographic parameters were compared with radiographic region localization, no significant correlation with multiple right ventricular dimensions was found.

\section{Discussion}

DX system is a unique technique in the era of ICDs. Until now, we have not had any data about periprocedural approach to optimization of AS. There are also no guidelines for patient selection for single-lead dual chamber ICD system. Our study investigated a probable relationship between floating atrial dipole position, as well as RA dimensions and AS in a population of patient who received single-lead dual chamber ICD. The major finding of our study is the negative correlation between RA size and the dependence of AS amplitude and floating dipole position in the RA.

Literature in the subject is very limited. Iori et al. [21] showed stable AS during 200-day follow-up but the population of the study was limited to only 13 patients. In the largest, so far, multicenter study including 116 patients with ICD DX system, Safak et al. [22] achieved appropriate AS in $93.8 \%$ of patients. In this study, mean preamplified P-wave amplitudes ranged from $5.0 \mathrm{mV}$ to $6.1 \mathrm{mV}$. In our set of patients, mean preamplified AS during the implantation procedure was $2.7 \mathrm{mV}(0.7-6.7 \mathrm{mV})$. During follow-up, in 1 patient, despite programing maximal sensitiv- 
ity, we did not achieve reliable AS and the atrial channel was turned off.

Supraventricular arrhythmias, mainly AF, are more common in subjects with HF and are associated with worse prognosis in this population $[5,6]$. Identification of patients at risk of future thromboembolic events associated with silent $\mathrm{AF}$ is a challenge. Use of monitoring of atrial electrical activity offers potential opportunity to identify such patients. In the ASSERT study, including patients with dual chamber pacemakers, it was demonstrated that subclinical AF of $\geq 6$ min duration was associated with 2.5-fold higher risk of stroke and systemic thromboembolism [23]. AF remains also the main cause of inappropriate therapies of ICDs [7, 8]. Proper AS plays a crucial role in both arrhythmia discrimination and supraventricular arrhythmia diagnostics. Inadequate AS may give inappropriate result of arrhythmia discrimination algorithm and trigger cardioversion delivery in case of supraventricular tachycardia. To receive adequate AS with floating dipole ICD it is advisable to use fourfold amplifier and special filtering of native signal. Enlargement of the atria was identified as a factor of higher prevalence of $\mathrm{AF}$ and lower likelihood of sinus rhythm restoration and maintenance [24, 25]. Former studies have shown that the size of atria is correlated with mean AS amplitude and AS stability in VDD pacemakers [26, 27]. This finding is consistent with our observations in this ICD population. It makes a paradoxical situation where the larger the atria the greater the risk of atrial undersensing and malfunction of the device. One cannot foresee the amplitude of f-wave during ICD implantation. Programing of achievably maximal sensitivity seems to be important in this population. In some patients during follow-up, loss of AS in device diagnostics may be suggestive for an $\mathrm{AF}$ episode.

Possible relationship between patients' height and AS amplitude was suggested [22]. In the present study, we did not find any connection between these variables. In our opinion, height may influence the choice of length of an electrode but should not have any significant impact on sensing.

Potentially useful techniques to achieve better AS owing to higher position of sensing dipole are the following: 1) using defibrillation leads with dipole located $17 \mathrm{~cm}$ from the tip of an electrode in enlarged hearts, 2) implantation of a lead to alternative places instead of right ventricle apex, and 3 ) playing with the course of an lead in RA (greater "heel" vs. more tense).

\section{Limitations of the study}

Our study has several limitations. First, the study population was relatively small, thus some analyses were underpowered to detect significant differences. We realize that results in a relatively small population sample may be significantly affected by a single measurement and there is a need for future investigation in a larger cohort of patients to better asses the relationships between atrial sensing and atrium diameter. Second, we analyzed only mean AS amplitude during normal breathing in lying position during the implantation procedure and in sitting position during predischarge and ambulatory follow-up. It is unknown whether results of AS test in different body positions would affect the results of our study. Third, standard chest $\mathrm{X}$-ray examination is performed in standing position during maximal inspiration, therefore the sensing dipole position may slightly differ in different circumstances.

\section{Conclusions}

Smaller dimensions of RA and higher position of floating dipole in the RA are predictors of better AS amplitude in single-lead dual chamber ICDs.

Conflict of interest: Marcin Michalak, Andrzej Cacko and Marcin Grabowski received educational grants from Biotronik Poland Sp. z o.o.

\section{References}

1. Carson P, Anand I, O'Connor C, et al. Mode of death in advanced heart failure: the Comparison of Medical, Pacing, and Defibrillation Therapies in Heart Failure (COMPANION) trial. J Am Coll Cardiol. 2005; 46(12): 2329-2334, doi: 10.1016/j. jacc.2005.09.016, indexed in Pubmed: 16360067.

2. Pons F, Lupón J, Urrutia A, et al. Mortality and cause of death in patients with heart failure: findings at a specialist multidisciplinary heart failure unit. Rev Esp Cardiol. 2010; 63(3): 303-314, doi: 10.1016/s1885-5857(10)70063-3, indexed in Pubmed: 20196991.

3. Priori S, Blomström-Lundqvist C, Mazzanti A, et al. 2015 ESC Guidelines for the management of patients with ventricular arrhythmias and the prevention of sudden cardiac death. Eur Heart J. 2015; 36(41): 2793-2867, doi: 10.1093/eurheartj/ehv316.

4. McMurray J, Adamopoulos S, Anker SD, et al. ESC Guidelines for the diagnosis and treatment of acute and chronic heart failure 2012: The Task Force for the Diagnosis and Treatment of Acute and Chronic Heart Failure 2012 of the European Society of Cardiology. Developed in collaboration with the Heart Failure Association (HFA) of the ESC. Eur Heart J. 2012; 33(14): 1787-1847, doi: 10.1093/eurheartj/ehs104. 
5. Kotecha D, Piccini JP. Atrial fibrillation in heart failure: what should we do? Eur Heart J. 2015; 36(46): 3250-3257, doi: 10.1093/eurheartj/ehv513, indexed in Pubmed: 26419625.

6. Mamas MA, Caldwell JC, Chacko S, et al. A meta-analysis of the prognostic significance of atrial fibrillation in chronic heart failure. Eur J Heart Fail. 2009; 11(7): 676-683, doi: 10.1093/eurjhf/ hfp085, indexed in Pubmed: 19553398.

7. Kutyifa V, Daubert JP, Olshansky B, et al. Characterization and predictors of first and subsequent inappropriate ICD therapy by heart rate ranges: Result of the MADIT-RIT efficacy analysis. Heart Rhythm. 2015; 12(9): 2030-2037, doi: 10.1016/j. hrthm.2015.05.021, indexed in Pubmed: 26001510.

8. Leenhardt A, Defaye P, Mouton E, et al. OPERA Registry Investigators. First inappropriate implantable cardioverter defibrillator therapy is often due to inaccurate device programming: analysis of the French OPERA registry. Europace. 2012; 14(10): 1465-1474, doi: 10.1093/europace/eus144, indexed in Pubmed: 22547767.

9. Berenbom LD, Weiford BC, Vacek JL, et al. Differences in outcomes between patients treated with single- versus dual-chamber implantable cardioverter defibrillators: a substudy of the Multicenter Automatic Defibrillator Implantation Trial II. Ann Noninvasive Electrocardiol. 2005; 10(4): 429-435, doi: 10.1111/j.1542474X.2005.00063.x, indexed in Pubmed: 16255753.

10. Bardy GH, Lee KL, Mark DB, et al. Amiodarone or an implantable cardioverter-defibrillator for congestive heart failure. N Engl J Med. 2005; 352(3): 225-237, doi: 10.1056/NEJMoa043399, indexed in Pubmed: 15659722.

11. Peterson PN, Varosy PD, Heidenreich PA, et al. Association of single- vs dual-chamber ICDs with mortality, readmissions, and complications among patients receiving an ICD for primary prevention. JAMA. 2013; 309(19): 2025-2034, doi: 10.1001/ jama.2013.4982, indexed in Pubmed: 23677314.

12. Dewland TA, Pellegrini CN, Wang Y, et al. Dual-chamber implantable cardioverter-defibrillator selection is associated with increased complication rates and mortality among patients enrolled in the NCDR implantable cardioverter-defibrillator registry. J Am Coll Cardiol. 2011; 58(10): 1007-1013, doi: 10.1016/j. jacc.2011.04.039, indexed in Pubmed: 21867834.

13. Friedman PA, Bradley D, Koestler C, et al. A prospective randomized trial of single- or dual-chamber implantable cardioverterdefibrillators to minimize inappropriate shock risk in primary sudden cardiac death prevention. Europace. 2014; 16(10): 1460-1468, doi: 10.1093/europace/euu022, indexed in Pubmed: 24928948.

14. Almendral J, Arribas F, Wolpert C, et al. Dual-chamber defibrillators reduce clinically significant adverse events compared with single-chamber devices: results from the DATAS (Dual chamber and Atrial Tachyarrhythmias Adverse events Study) trial. Europace. 2008; 10(5): 528-535, doi:10.1093/europace/eun072, indexed in Pubmed: 18390985.

15. Theuns DA, Rivero-Ayerza M, Boersma E, et al. Prevention of inappropriate therapy in implantable defibrillators: A metaanalysis of clinical trials comparing single-chamber and dualchamber arrhythmia discrimination algorithms. Int J Cardiol. 2008; 125(3): 352-357, doi:10.1016/j.ijcard.2007.02.041, indexed in Pubmed: 17445918.
16. Sinha AM, Stellbrink C, Schuchert A, et al. Clinical experience with a new detection algorithm for differentiation of supraventricular from ventricular tachycardia in a dual-chamber defibrillator. J Cardiovasc Electrophysiol. 2004; 15(6): 646-652, doi: 10.1046/j.1540-8167.2004.03290.x, indexed in Pubmed: 15175058.

17. Gold MR, Ahmad S, Browne K, et al. Prospective comparison of discrimination algorithms to prevent inappropriate ICD therapy: primary results of the Rhythm ID Going Head to Head Trial. Heart Rhythm. 2012; 9(3): 370-377, doi: 10.1016/j. hrthm.2011.10.004, indexed in Pubmed: 21978966.

18. Glikson M, Swerdlow CD, Gurevitz OT, et al. Optimal combination of discriminators for differentiating ventricular from supraventricular tachycardia by dual-chamber defibrillators. J Cardiovasc Electrophysiol. 2005; 16(7): 732-739, doi: 10.1046/j.15408167.2005.40643.x, indexed in Pubmed:16050831.

19. Rudski LG, Lai WW, Afilalo J, et al. Guidelines for the echocardiographic assessment of the right heart in adults: a report from the American Society of Echocardiography endorsed by the European Association of Echocardiography, a registered branch of the European Society of Cardiology, and the Canadian Society of Echocardiography. J Am Soc Echocardiogr. 2010; 23(7): 685-713; quiz 786, doi: 10.1016/j.echo.2010.05.010, indexed in Pubmed:20620859.

20. LANG R, BIERIG M, DEVEREUX R, et al. Recommendations for chamber quantification. Eur J Echocardiogr. 2006; 7(2): 79108, doi:10.1016/j.euje.2005.12.014.

21. Iori M, Giacopelli D, Quartieri F, et al. Implantable cardioverter defibrillator system with floating atrial sensing dipole: a single-center experience. Pacing Clin Electrophysiol. 2014; 37(10): 1265-1273, doi: 10.1111/pace.12421, indexed in Pubmed: 24809851.

22. Safak E, Schmitz D, Konorza T, et al. Linox DX Study Investigators. Clinical efficacy and safety of an implantable cardioverterdefibrillator lead with a floating atrial sensing dipole. Pacing Clin Electrophysiol. 2013; 36(8): 952-962, doi: 10.1111/pace.12171, indexed in Pubmed: 23692262.

23. Healey J, Connolly S, Gold M, et al. Subclinical Atrial Fibrillation and the Risk of Stroke. New Engl J Med. 2012; 366(2): 120-129, doi:10.1056/nejmoa1105575.

24. Vaziri SM, Larson MG, Benjamin EJ, et al. Echocardiographic predictors of nonrheumatic atrial fibrillation. The Framingham Heart Study. Circulation. 1994; 89(2): 724-730, doi: 10.1161/01. cir.89.2.724.

25. ASINGER R. Role of Transthoracic Echocardiography in Atrial Fibrillation. Echocardiography. 2000; 17(4): 357-364, doi: 10.1111/j.1540-8175.2000.tb01151.x.

26. Santini M, Ricci R, Pignalberi C, et al. Immediate and longterm atrial sensing stability in single-lead VDD pacing depends on right atrial dimensions. Europace. 2001; 3(4): 324-331, doi: 10.1053/eupc.2001.0188, indexed in Pubmed: 11678392.

27. de Cock CC, Van Campen LC, Huygens J, et al. Usefulness of echocardiography to predict inappropriate atrial sensing in single-lead VDD pacing. Pacing Clin Electrophysiol. 1999; 22(9): 1344-1347, doi: 10.1111/j.1540-8159.1999.tb00627.x, indexed in Pubmed: 10527015. 